\title{
Complete remission of metastatic osteosarcoma using combined modality therapy: a retrospective analysis of unselected patients in China
}

Lu Xie ${ }^{1}$, Jie Xu ${ }^{1}$, Xiaowei Li ${ }^{1}$, Zuli Zhou ${ }^{2}$, Hongqing Zhuang ${ }^{3}$, Xin Sun ${ }^{1}$, Kuisheng Liu' ${ }^{1}$ Xingyu Liu ${ }^{1}$, Kunkun Sun $^{4}$, Yiming $\mathrm{Wu}^{5}$, Jin $\mathrm{Gu}^{6}$ and Wei Guo ${ }^{1 *}$ (i)

\begin{abstract}
Background: Complete surgical remission (CSR) is the best predictor of overall survival (OS) for patients with metastatic osteosarcoma. However, metastasectomy has not been widely implemented in China in the last decade due to various factors, and instead, most physicians choose hypofractionated radiotherapy to treat pulmonary lesions. This study aimed to retrospectively evaluate the outcomes of different local treatments for pulmonary lesions and identify the best local therapy strategies for these patients.

Methods: We reviewed the clinical courses of osteosarcoma patients with pulmonary metastases who were initially treated in two sarcoma centres in Beijing, China, from June 1st, 2009, to March 26th, 2020. With a median follow-up of 32.4 (95\% confidence interval (Cl): 30.8, 36.1) months, a total of 127 patients with 605 pulmonary nodules, all of whom had received local therapy and firstly achieved CSR or complete radiated/metabolic remission (CRR), were included in the analysis. A total of 102 patients with 525 nodules were initially diagnosed with resectable lung metastases, while 25 patients had 80 indeterminate nodules at presentation and relapsed with pulmonary metastases within 6 months after the completion of adjuvant chemotherapy.
\end{abstract}

Results: Eighty-eight of 127 (69.3\%) patients had fewer than 5 nodules at the time of local therapy, with 48 of 127 (37.8\%) located in the unilateral pleura. No patient underwent thoracotomy, and 42 of 127 patients (85 nodules) received video-assisted thoracoscopic surgery (VATS). In addition, 79 of 127 patients (520 nodules) received hypofractionated stereotactic body radiotherapy (RT), such as Gamma Knife radiosurgery or CyberKnife radiosurgery. The twelve-month event-free survival (EFS) (from local therapy to progression) rate of this entire study cohort was 35.6\% (95\% Cl: 26.8, 44.4\%), without a significant difference between the two groups (44.7\% for VATS vs. 28.4\% for $\mathrm{RT}, P=0.755)$. Radiation-induced pneumonitis was observed in 62 of $86(72.1 \%)$ patients, with one patient $(1 / 86$, $1.2 \%)$ in grade 4.

* Correspondence: bonetumor@163.com

${ }^{1}$ Musculoskeletal Tumor Center, Peking University People's Hospital, No. 11 Xizhimen South Street, Xicheng District, Beijing, PR China

Full list of author information is available at the end of the article

(C) The Author(s). 2021 Open Access This article is licensed under a Creative Commons Attribution 4.0 International License, which permits use, sharing, adaptation, distribution and reproduction in any medium or format, as long as you give appropriate credit to the original author(s) and the source, provide a link to the Creative Commons licence, and indicate if changes were made. The images or other third party material in this article are included in the article's Creative Commons licence, unless indicated otherwise in a credit line to the material. If material is not included in the article's Creative Commons licence and your intended use is not permitted by statutory regulation or exceeds the permitted use, you will need to obtain permission directly from the copyright holder. To view a copy of this licence, visit http://creativecommons.org/licenses/by/4.0/ The Creative Commons Public Domain Dedication waiver (http://creativecommons.org/publicdomain/zero/1.0/) applies to the data made available in this article, unless otherwise stated in a credit line to the data. 
(Continued from previous page)

Conclusions: Our past data showed a similar prognosis with the use of hypofractionated radiotherapy and VATS for the treatment of pulmonary metastasis and no inferiority to thoracotomy regarding historical outcomes. Currently, high-resolution chest computed tomography (CT) provides sufficient information on nodules, and less invasive modalities can thus be considered for treatment.

Keywords: Osteosarcoma, Pulmonary metastasis, Hypofractionated radiotherapy, Event-free survival, Radiation pneumonitis

\section{Background}

Patients who develop pulmonary metastasis from osteosarcoma usually have a relatively poor prognosis, with a 5 -year overall survival (OS) rate of $20-40 \%$; those who initially present with countable pulmonary lesions have a 5 -year OS rate of approximately $40 \%$, whereas those with recurrent osteosarcoma have a 5 -year OS rate of $28 \%$ or even less [1-3]. The most significant prognosis is the achievement of complete remission [4]. Therefore, the goal of local remission of pulmonary metastases is to render the patient completely disease free. "Tumour debulking" or "cytoreductive surgery" with incomplete resection has not demonstrated any survival benefit. Nevertheless, the timing of local therapy as well as local therapeutic methods remains controversial [5]. To date, thoracotomy has been accepted worldwide for resecting palpable lesions regardless of whether these nodules are present on chest computed tomography (CT) [6, 7]. However, with the development of high-resolution chest CT (with $<3 \mathrm{~mm}$ per layer), we aimed to determine whether certain nodules would still be missed on imaging. Can other less invasive local treatment methods replace open resection of pulmonary lesions?

In the last decade, thoracotomy has not been widely performed in China due to numerous reasons. First and most importantly, it has not been realized by the majority of thoracic surgeons that complete surgical remission (CSR) of osteosarcoma plays an important role in its prognosis. Second, although video-assisted thoracoscopic surgery (VATS) has been widely adopted for other pulmonary diseases, it is rarely accepted that doctors perform thoracotomies only for metastatic resection of osteosarcoma. Third, multidisciplinary teamwork (MDT), especially for orthopaedic oncologists, medical oncologists, and thoracic surgeons, is not enough for sarcoma treatment in China. Thus, we rarely perform both orthopaedic and thoracic surgeries together under a single anaesthetic.

Stereotactic body radiotherapy (SBRT) is a theoretically attractive local treatment modality in certain patients with advanced osteosarcoma [8], as it is convenient, minimizes delays in chemotherapy administration, and offers the possibility of increased efficacy via biologically effective dose escalation. Gamma Knife radiosurgery (GKRS) has proven to be a low-risk and effective treatment strategy for a wide variety of patients with brain metastases $[9,10]$. In addition, there are a growing number of reports describing the use of an SBRT boost by a robotic stereotactic system (CyberKnife) to an average additional dose of $25 \mathrm{~Gy}$ (range: 20-30 Gy) to treat pulmonary lesions in a variety of tumours [11]. Thus, we tried to use GKRS or CyberKnife radiosurgery to treat osteosarcoma pulmonary metastasis in the past. This study aimed to summarize our experience in treating pulmonary metastatic osteosarcoma and compare the outcomes to published historical data abroad to discuss the optimal local therapeutic method for these lesions.

\section{Methods}

From June 1st, 2009, to March 26th, 2020, 973 consecutive patients histologically diagnosed with high-grade osteosarcoma were initially treated at Peking University People's Hospital (PKUPH) and Peking University Shougang Hospital in Beijing, China. Both hospitals obtained institutional review board approval to review the patients' medical records as well as radiographic materials. Their outcome data were then retrospectively combined. Written informed consent was not required.

The diagnosis of high-grade osteosarcoma, all established by pathologists using musculoskeletal tumour tissues, was always confirmed and reviewed on histologic slides obtained from resected specimens by two senior pathologists at PKUPH. We included all patients with pulmonary metastasis at the initial presentation, some of whom initially presented with "possible" metastasis according to the EURAMOS-1 protocol [12] and then developed "certain" pulmonary metastasis, with the intention of eradicating all pulmonary nodules. This allowed us to examine the data from 127 patients (605 lesions), all of whom were undergoing surgery or receiving radiation to achieve CSR or complete radiated/metabolic remission (CRR) for the first time.

For high-grade osteosarcomas with pulmonary nodules, we recorded those with the largest diameter $\left(D_{\max }\right)$ of more than $3 \mathrm{~mm}$. However, those with solitary nodules ( $\geq 1 \mathrm{~cm}$ in diameter) required pathological confirmation by resection, while those with multiple pulmonary 
nodules $\left(3 \mathrm{~mm} \leq \mathrm{D}_{\max } \leq 5 \mathrm{~mm}\right.$ ) were always followed up to confirm their development into metastasis. We recorded the initial time at which these nodules were detected by high-resolution chest CT, which was always assessed by spiral CT scanning, with each layer less than $3 \mathrm{~mm}$. Thoracic surgeons or radiologists always determined whether these pulmonary nodules were resectable based on each individual's circumstances. Usually, we excluded patients with more than 10 nodules, which might cause severe radiation-induced pneumonitis, from hypofractionated radiotherapy. However, for thoracic surgeries, patients with more than 5 lesions and nodules located at central zones of the lung were also excluded from resection. Specifically, according to radiologists, GKRS was not performed on lesions more than $20 \mathrm{~mm}$ in size; however, for CyberKnife radiosurgery, nodule size was not stringently considered a limitation.

The 127 patients enrolled in this study had evident lung metastases based on pathology or long-term followup, 102 of whom had certain metastasis at the initial diagnosis, while 25 had indeterminate pulmonary nodules that later developed into certain metastasis. Local therapy was always performed upon effective systemic treatment (i.e., partial response or stable disease based on the RECIST 1.1). However, patients with slow, longterm (6 months or longer) tumour growth were also considered for local eradication. A total of 605 nodules were observed, 85 of which received VATS and 520 of which received RT. With a median follow-up time of 32.4 (95\% confidence interval $(\mathrm{CI})$ : $30.8,36.1)$ months, 110 of $127(86.6 \%)$ patients survived, and 17 of 127 (13.4\%) died of disease. Thus, we selected event-free survival (from VATS or RT to any event related to disease progression) rather than overall survival (OS, from VATS or RT to death) to analyse patient outcomes.

During systemic treatment, patients underwent highresolution chest $\mathrm{CT}$ and radiography of the operated musculoskeletal sites every 2 months. After the completion of local therapy, patients were also followed every 2 months for the first 2 years and then every 3 months for the next 3 years. Radionuclide bone scans or positron emission tomography (PET)/CT was used to assess metastatic disease every 6 months for the first 5 years after the completion of systemic treatment.

For this study, event-free survival (EFS) was calculated from the local eradication of pulmonary lesions (resection or radiation) until any event related to disease progression or death, whichever came first. We calculated OS from the time of local therapy to death from any cause. Demographic data are displayed as descriptive statistics. Kaplan-Meier analysis was employed to determine OS, EFS, and recurrence-free survival (RFS). Cox proportional hazards analysis was subsequently performed on variables to identify factors associated with survival and local recurrence. Differences with a $P$ value $<0.05$ were considered statistically significant. All analyses were performed using SPSS 19.0 software (SPSS Inc., Chicago, IL, USA).

\section{Results}

Patient demographics are summarized in Table 1. At the initial diagnosis, the median age of all eligible patients was 15.0 years (range: 5.0 to 56.0 years). One hundred and fifteen osteosarcomas (90.6\%) were situated in the extremities, and 7 osteosarcomas (5.5\%) were in the axial skeleton. One osteosarcoma originated from the maxillofacial site, and two originated from the ribs. Eighty-eight of $127(69.3 \%)$ patients had < 5 pulmonary nodules, and 48 of 127 (37.8\%) had nodules that were unilaterally located. Based on the $\mathrm{D}_{\max }$ of the largest pulmonary nodule of each individual treated during local therapy, patients were classified into four categories: $3-5 \mathrm{~mm}$ (18/127, 14.2\%), 5-10 mm (29/127, 22.8\%), 10-20 mm (50/127, 39.4\%), and $>20 \mathrm{~mm}$ (30/127, 23.6\%). A total of $80.3 \%(102 / 127)$ of these patients had synchronous pulmonary metastasis at diagnosis, while $19.7 \%$ (25/127) had uncertain pulmonary nodules during the initial diagnosis according to the EURAMOS-1 protocol [12]; additionally, nearly all uncertain pulmonary nodules developed into certain metastases within 6 months after the termination of first-line chemotherapy (Appendix Fig. 1).

At the time of local therapy, the median maximum diameter $\left(D_{\max }\right)$ of the treated lesion was 15.8 (IQR, 7.5, $22,4) \mathrm{mm}$. For radiation, the gross target volume (GTV) was calculated for image-visible lesions based on 1.5mm slice thickness chest $\mathrm{CT}$, and $70 \sim 85 \%$ isodose lines covered the target areas of the planning target volume (PTV). The PTVs were radiated based on the GTVs with reference to the $4 \mathrm{D}-\mathrm{CT}$ images, and the targets were divided according to the volume and position of the lesions. For CyberKnife radiosurgery, 24/30 Gy/1 f (24 or $30 \mathrm{~Gy}$ ) was given to lesions that were not close to the mediastinum and chest wall, $45 \mathrm{~Gy} / 3 \mathrm{f}$ was given to lesions close to the chest wall and mediastinum, and 36 $\mathrm{Gy} / 3 \mathrm{f}$ was given to lesions close to the hilum and bronchial tree. Non-isocentre non-coplanar radiation technology was used to design the treatment plan, and stereotactic radiotherapy was performed. A median dose of 26 (IQR, 20, 33) Gy was delivered to these lesions.

Fifty-six of 127 (44.1\%) patients underwent tumour resection or received radiation during first-line chemotherapy following the PKUPH-OS regimen (Appendix Fig. 1), which included high-dose methotrexate, doxorubicin, cisplatin, and ifosfamide. Fifty-three of 127 $(41.7 \%)$ patients were stabilized upon second-line chemotherapy, which included ifosfamide $\left(1.8 \mathrm{~g} / \mathrm{m}^{2} / \mathrm{d}\right.$ $\left.\mathrm{d}_{1-5}\right)$ and etoposide $\left(100 \mathrm{mg} / \mathrm{m}^{2} / \mathrm{d} \mathrm{d}_{1-5}\right.$ Q3W), most of 
Table 1 Patient characteristics $(N=127)$

\begin{tabular}{|c|c|c|c|}
\hline Items & Number of patients & Percentage (\%) & $p$ for 2-y EFS \\
\hline Sex & & & 0.759 \\
\hline Male & 83 & 65.4 & \\
\hline Female & 44 & 34.7 & \\
\hline Age (median: 15.0 years) & \multicolumn{2}{|c|}{ Range: 5-56 (Q1, Q3, 15.1, 18.0) years } & 0.025 \\
\hline$<40$ years & 124 & 97.6 & \\
\hline$\geq 40$ years & 3 & 2.4 & \\
\hline Pathological Subtypes & & & 0.526 \\
\hline Conventional:chondroblastic & 12 & 9.4 & \\
\hline Conventional: osteoblastic & 78 & 61.4 & \\
\hline Conventional: not defined & 24 & 18.9 & \\
\hline Telangiectatic & 5 & 3.9 & \\
\hline Small cell & 3 & 2.4 & \\
\hline High-grade surface & 1 & 0.8 & \\
\hline Missing & 4 & 3.1 & \\
\hline Primary site & & & 0.328 \\
\hline Distal femur & 62 & 48.8 & \\
\hline Proximal tibia and/or fibula & 38 & 29.9 & \\
\hline Proximal femur & 4 & 3.2 & \\
\hline Proximal humerus & 9 & 7.1 & \\
\hline Axial skeleton & 7 & 5.5 & \\
\hline Maxillofacial site & 1 & 0.8 & \\
\hline Others & 6 & 4.7 & \\
\hline Total number of pulmonary nodules for observation & 605 & 100 & 0.963 \\
\hline Lung metastasis & & & 0.464 \\
\hline$\leq 5$ nodules & 88 & 69.3 & \\
\hline$>5$ nodules & 39 & 30.7 & \\
\hline Lung metastasis & & & 0.063 \\
\hline Monolateral & 48 & 37.8 & \\
\hline Bilateral & 79 & 62.2 & \\
\hline Local therapy for pulmonary nodules & & & 0.476 \\
\hline Resection $^{\mathrm{b}}$ & 42 (85 nodules) & 33.1 & \\
\hline Radiotherapy ${ }^{c}$ & 79 (520 nodules) & 62.2 & \\
\hline Combined with resection and radiotherapy & 6 & 4.7 & \\
\hline Missing nodules during follow-up ${ }^{d}$ & 52 & $7.9(52 / 657)$ & $N / A^{i}$ \\
\hline For resection & 15 & $15.0(15 / 100)$ & \\
\hline For radiotherapy & 37 & $6.6(37 / 557)$ & \\
\hline Failed local resection ${ }^{\mathrm{e}}$ & 8 & $9.4(8 / 85)$ & \\
\hline Failed local radiotherapy ${ }^{f}$ & 14 & $2.7(14 / 520)$ & \\
\hline$D_{\max }{ }^{9}$ for pulmonary nodule/nodules & & & 0.286 \\
\hline $3-5 \mathrm{~mm}$ & 18 & 14.1 & \\
\hline $5-10 \mathrm{~mm}$ & 29 & 22.8 & \\
\hline $10-20 \mathrm{~mm}$ & 50 & 39.4 & \\
\hline$>20 \mathrm{~mm}$ & 30 & 23.6 & \\
\hline Systematic treatment during local therapy of pulmonary & & & 0.426 \\
\hline
\end{tabular}


Table 1 Patient characteristics $(N=127)$ (Continued)

\begin{tabular}{llll}
\hline Items & Number of patients & Percentage (\%) & p for 2-y EFS \\
\hline MAPI first-line chemotherapy & 56 & 44.1 & \\
IE second-line chemotherapy & 53 & 41.7 \\
Targeted therapy & 13 & 10.2 \\
Combination of TKIs and IE chemo & 4 & 3.2 \\
None & 1 & 0.8 & $(95 \%$ Cl: 30.8, 36.1) \\
Median time for follow-up (months) & 32.4 & (Range: 10.4, 106.5)
\end{tabular}

a 2-y EFS: 2-year event-free survival, which was calculated from start of the local therapy (resection or radiotherapy) to any kind of progression as defined by RECIST 1.1

${ }^{\mathrm{b}}$ Pulmonary metastasectomies were video-assisted thoracoscopic Surgery (VATS)

'Radiotherapy usually involves GammaKnife or Cyber Knife with radio-dose $>60$ Gy

${ }^{d}$ By comparing initial chest thin-layer computed tomography $(C T)$ before local therapy and during follow-up, we observed that nodules had resolved or were undetectable with local treatment, most of which were observed as tiny or blurry nodules or even hardly been detected between infection and malignancy and would relapse after stopping systemic treatment

${ }^{\mathrm{e}}$ Failed local resection: local tumor relapse where previous tumor resection had been done

${ }^{f}$ Failed local radiotherapy: local tumor relapse where previous radiation had been performed for curative tumor eradication

${ }^{9}$ Patients were classified into four groups based on maximal nodule diameter: 1) $3 \mathrm{~mm}-5 \mathrm{~mm}$; 2) $5 \mathrm{~mm}-10 \mathrm{~mm}$; 3) $10 \mathrm{~mm}-20 \mathrm{~mm}$; 4 ) $20 \mathrm{~mm}$

${ }^{h}$ At the Musculoskeletal Tumor Center of Peking University People's Hospital and Peking University Shougang Hospital, a chemo-protocol that includes high-dose methotrexate, cisplatin, doxorubicin, and ifosfamide (MAPI) is used as first-line chemotherapy (seen in appendix Fig. 1); ifosfamide and etoposide (IE) as second-

line systematic therapy; anti-angiogenesis tyrosine kinase inhibitors (TKIs) such as apatinib, anlotinib, cabozantinib, and regorafenib as third-line therapy; the

combination of TKIs and IE chemotherapy as fourth-line therapy

iData not available

who were initially diagnosed with indeterminate pulmonary nodules. We eradicated metastases in 13 of 127 (10.2\%) patients upon stable disease using antiangiogenetic tyrosine kinase inhibitors (TKIs), such as apatinib and regorafenib, while 4 of 127 (3.2\%) received a combination of TKIs and chemotherapy. One patient did not receive any systemic therapy because he had indeterminate pulmonary nodules at presentation and slow tumour growth after the completion of chemotherapy and refused to receive any additional chemotherapy peri-operatively.

In the univariate analysis, we compared sex, age, pathological subtypes, primary tumour site, timing of presentation with pulmonary lesions, nodule number, location in the unilateral pleura, local therapeutic methods, maximum diameter of the lesion treated, and lines of systemic therapy during local therapy, of which only age significantly influenced EFS. However, we analysed only 3 patients older than 40 years; therefore, these results may be inconclusive based on the disparity in the population. After controlling for all these confounders for the multivariate analysis, none remained independent factors associated with EFS, which implied us that the prognosis might not be different only if we got the way to get rid of all the metastatic lesions and tumor number or locations might not influence the local therapeutic efficacy for radiotherapy if we had planned well.

For the past few years, what kinds of local treatments have most Chinese patients with resectable pulmonary metastasis selected?

Forty-two of 127 (33.1\%) patients with 85 nodules chose to undergo resection via VATS. No one had ever received thoracotomy, partly because of the physician's preference and partly because of a lack of knowledge of thoracotomy or because of fear of trauma. Seventy-nine of 127 (62.2\%) patients received RT to eradicate 520 nodules.

Was there selection bias in choosing between pulmonary metastasectomy and radiation?

A comparison of the clinical and pathological factors of the two groups of patients is presented in Table 2 . Forty-one of $42(97.6 \%)$ patients who underwent VATS had $<5$ pulmonary nodules, while only 55 of 79 (69.6\%) patients who received RT had $<5$ pulmonary nodules. In addition, six patients received both RT and VATS. For lesions that was difficult to access during VATS, surgeons marked the lesions on chest $\mathrm{CT}$ and advised patients to receive RT 2 weeks after surgery. Patients with bilateral metastasis chose RT more than VATS. VATS was generally chosen by patients with a maximal lesion diameter between $10 \mathrm{~mm}$ and $20 \mathrm{~mm}(24 / 42,57.1 \%)$. For radiation, patients were almost equally distributed among different size groups. However, it seemed that patients who received VATS had more indeterminate pulmonary nodules at presentation, most of whom needed pathological confirmation through surgery.

\section{Did different local therapy methods influence patient outcomes?}

Until the last follow-up, local relapse after VATS occurred in $9.4 \%(8 / 85)$ of patients, while local recurrence after RT occurred in $2.7 \%(14 / 520)$ of patients (Table 3$)$. The 2-year non-local recurrence survival rates [SD] were 81.0\% [7.1\%] and 92.8\% [3.1\%] for resection and 
Table 2 Comparison of clinical manifestations of patients who underwent VATS ${ }^{a}$ or radiation

\begin{tabular}{|c|c|c|c|}
\hline Items & VATS $(N=42)$ & Radiotherapy $(N=79)$ & Combination $(N=6)$ \\
\hline \multicolumn{4}{|l|}{ Number of pulmonary nodules /person } \\
\hline$\leq 5$ nodules & $41(97.6 \%)$ & $55(69.6 \%)$ & $4(66.7 \%)$ \\
\hline$>5$ nodules & $1(2.4 \%)$ & $24(30.4 \%)$ & $2(33.3 \%)$ \\
\hline \multicolumn{4}{|l|}{ Lung metastasis } \\
\hline Monolateral & $33(78.6 \%)$ & $11(13.9 \%)$ & $4(66.7 \%)$ \\
\hline Bilateral & $9(21.4 \%)$ & $68(86.1 \%)$ & $2(33.3 \%)$ \\
\hline \multicolumn{4}{|l|}{$D_{\max }{ }^{b}$ for pulmonary nodule/nodules } \\
\hline $3-5 \mathrm{~mm}$ & $3(7.1 \%)$ & $15(19.0 \%)$ & $0(0.0 \%)$ \\
\hline $5-10 \mathrm{~mm}$ & $6(14.3 \%)$ & $22(27.8 \%)$ & $1(16.7 \%)$ \\
\hline $10-20 \mathrm{~mm}$ & $24(57.1 \%)$ & $25(31.6 \%)$ & $1(16.7 \%)$ \\
\hline$>20 \mathrm{~mm}$ & $9(21.4 \%)$ & $17(21.5 \%)$ & $4(66.7 \%)$ \\
\hline \multicolumn{4}{|c|}{ Systematic treatment during local therapy of pulmonary nodues ${ }^{c}$} \\
\hline MAPI first-line chemotherapy & $14(33.3 \%)$ & $40(50.6 \%)$ & $2(33.3 \%)$ \\
\hline IE second-line chemotherapy & $24(57.1 \%)$ & $25(31.6 \%)$ & $4(66.7 \%)$ \\
\hline Targeted therapy & $2(4.8 \%)$ & $11(13.9 \%)$ & $0(0.0 \%)$ \\
\hline Combination of TKIS and IE chemo & $2(4.8 \%)$ & $4(5.1 \%)$ & $0(0.0 \%)$ \\
\hline None & $0(0.0 \%)$ & $1(1.3 \%)$ & $0(0.0 \%)$ \\
\hline
\end{tabular}

${ }^{a}$ VATS Video-assisted thoracoscopic surgery

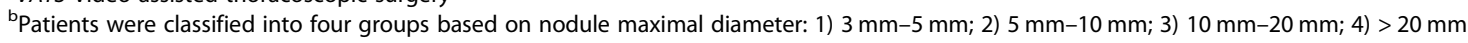

'At the Musculoskeletal Tumor Center of Peking University People's Hospital and Peking University Shougang Hospital, a chemo-protocol that includes high-dose methotrexate, cisplatin, doxorubicin, and ifosfamide (MAPI) is used as first-line chemotherapy (seen in appendix Fig. 1); ifosfamide and etoposide (IE) as secondline systematic therapy; anti-angiogenesis tyrosine kinase inhibitors (TKIs) such as apatinib, anlotinib, cabozantinib, and regorafenib as third-line therapy; and the combination of TKIs and IE chemotherapy as fourth-line therapy

radiation, respectively $(P=0.37)$. The median EFS times were 10.0 (IQR, 4.1, 17.1) and 10.1 (IQR, 5.8, 14.5) months for VATS and RT, respectively, without a significant difference $(P=0.76)$ (Figs. 1 and 2).

A review of all these high-resolution chest CT scans revealed that numerous nodules were missed (Figs. 3, 4, 5 and 6) before local therapy regardless of whether the patients received VATS or RT. For 85 resected nodules, high-resolution chest CT 2 weeks after surgery (we usually performed a scan shortly after surgery in the case of pneumothorax or haemothorax to deliver postoperative systemic therapy) showed 15 nodules missing (17.7\%, all $<5 \mathrm{~mm}$ ) that later grew larger in size and were confirmed as metastasis clinically or pathologically. For radiation, by comparing the chest $\mathrm{CT}$ scan before RT with targetplanning photographs for RT, we noticed that 7.12\% (37/ 520 ) of the nodules were missed for eradication and later progressed. Detailed information is presented in Table 3. In addition, we observed that the majority of cases of disease progression were not accompanied by local relapse. No correlation between local relapse and disease progression was detected $(P=0.37)$.

Table 3 Comparison of survival in different groups of patients

\begin{tabular}{|c|c|c|c|c|}
\hline Items & $\begin{array}{l}\text { Patients with } \\
\text { Resections } \\
(N=42)\end{array}$ & $\begin{array}{l}\text { Patients with } \\
\text { Radiotherapy } \\
(N=79)\end{array}$ & $\begin{array}{l}\text { Patients with combination } \\
\text { of resections and radiotherapy } \\
(N=6)\end{array}$ & $\begin{array}{l}p \text { for } \\
\text { survival }\end{array}$ \\
\hline 2-year no local recurrence survival rate $[ \pm S D]$ & $81.0 \%[ \pm 7.1 \%]$ & $92.8 \%[ \pm 3.1 \%]$ & $66.7 \%[ \pm 36.7 \%]$ & 0.652 \\
\hline Local relapse of nodules without new lesions & $2 / 42(4.8 \%)$ & $1 / 79(1.3 \%)$ & $1 / 6(16.7 \%)$ & $N / A^{a}$ \\
\hline Local relapse of nodules with new lesions & $6 / 42(14.3 \%)$ & $10 / 79(12.7 \%)$ & $1 / 6(16.7 \%)$ & $N / A^{a}$ \\
\hline Progression without local relapse & 19/42 (45.2\%) & $49 / 79(62.0 \%)$ & $3 / 6(50.0 \%)$ & \\
\hline Events for progression in total & $27 / 42(64.3 \%)$ & $60 / 79(75.9 \%)$ & $5 / 6(83.3 \%)$ & \\
\hline From resections/radiotherapy to any event (median, Q1, Q3) months & $10.0(4.1,17.1)$ & $10.1(5.8,14.5)$ & N/A & 0.755 \\
\hline From resections/radiotherapy to death (mean, $95 \% \mathrm{Cl})^{\mathrm{b}}$ months & $37.6(32.5,42.7)$ & $67.0(58.7,75.3)$ & $21.5(17.3,25.7)$ & 0.712 \\
\hline
\end{tabular}

${ }^{a} \mathrm{~N} / \mathrm{A}$ data not available

${ }^{b}$ median overall survival has not reached yet, thus we use mean overall survival to replace the data 


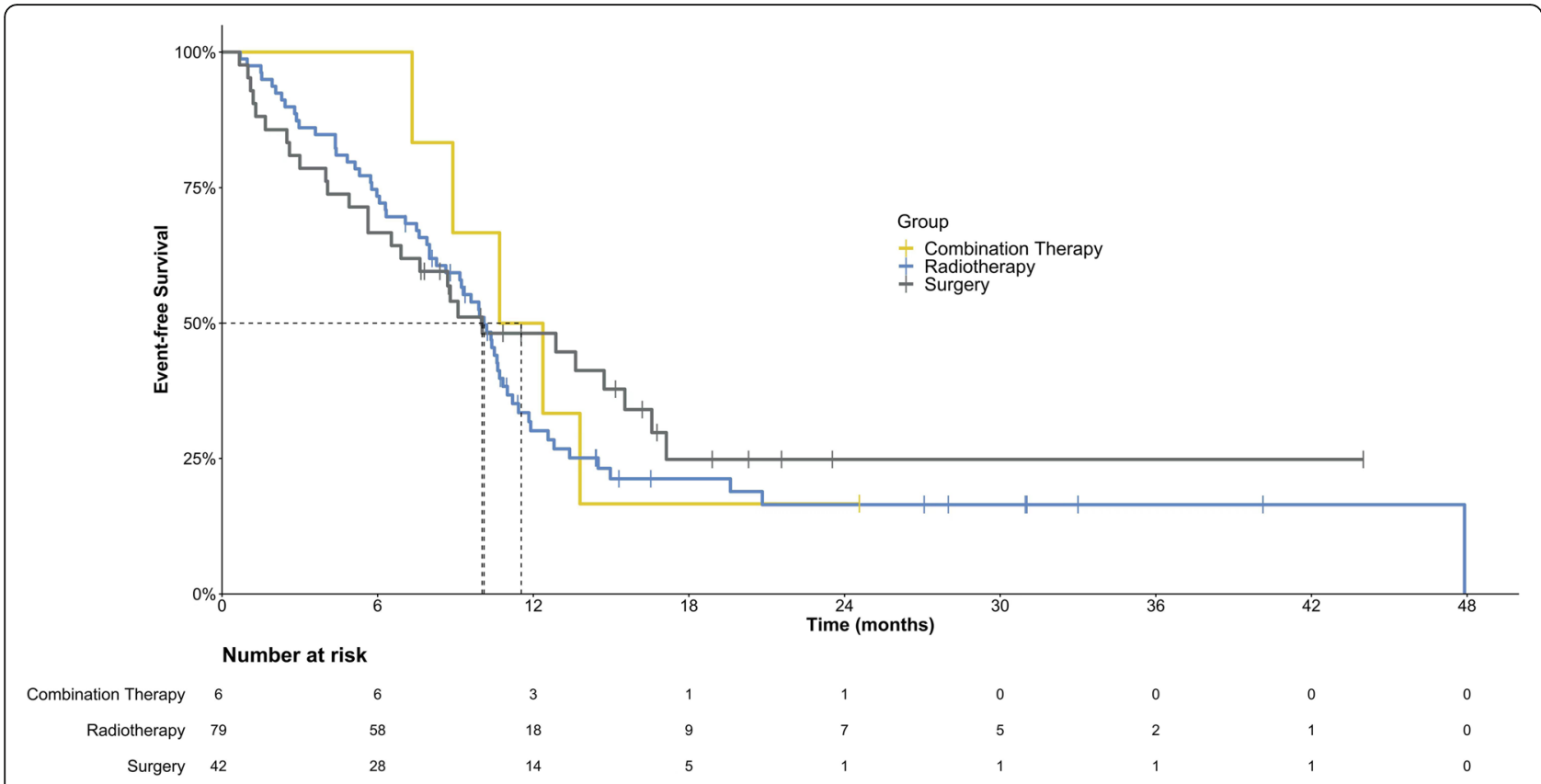

Fig. 1 Kaplan-Meier plot for event-free survival in all 127 patients

How do the data of the present study compare to those of published historical controls?

In total, these patients showed a 12-month EFS rate of $35.6 \%$ (95\% CI: $26.8,44.4 \%$ ), which was concordant with the findings of the Children's Oncology Group (20 to $31 \%$ in the prospective trials of AOST1221 [13] and
AOST1421 [14]). For patients who received VATS, the 12-month EFS rate was $44.7 \%$, whereas for patients who received $\mathrm{RT}$, it was 28.4\%; however, this difference was not statistically significant ( $P=0.76$, Fig. 2 ). Our findings on OS should be considered preliminary. No obvious difference in prognosis was observed between the

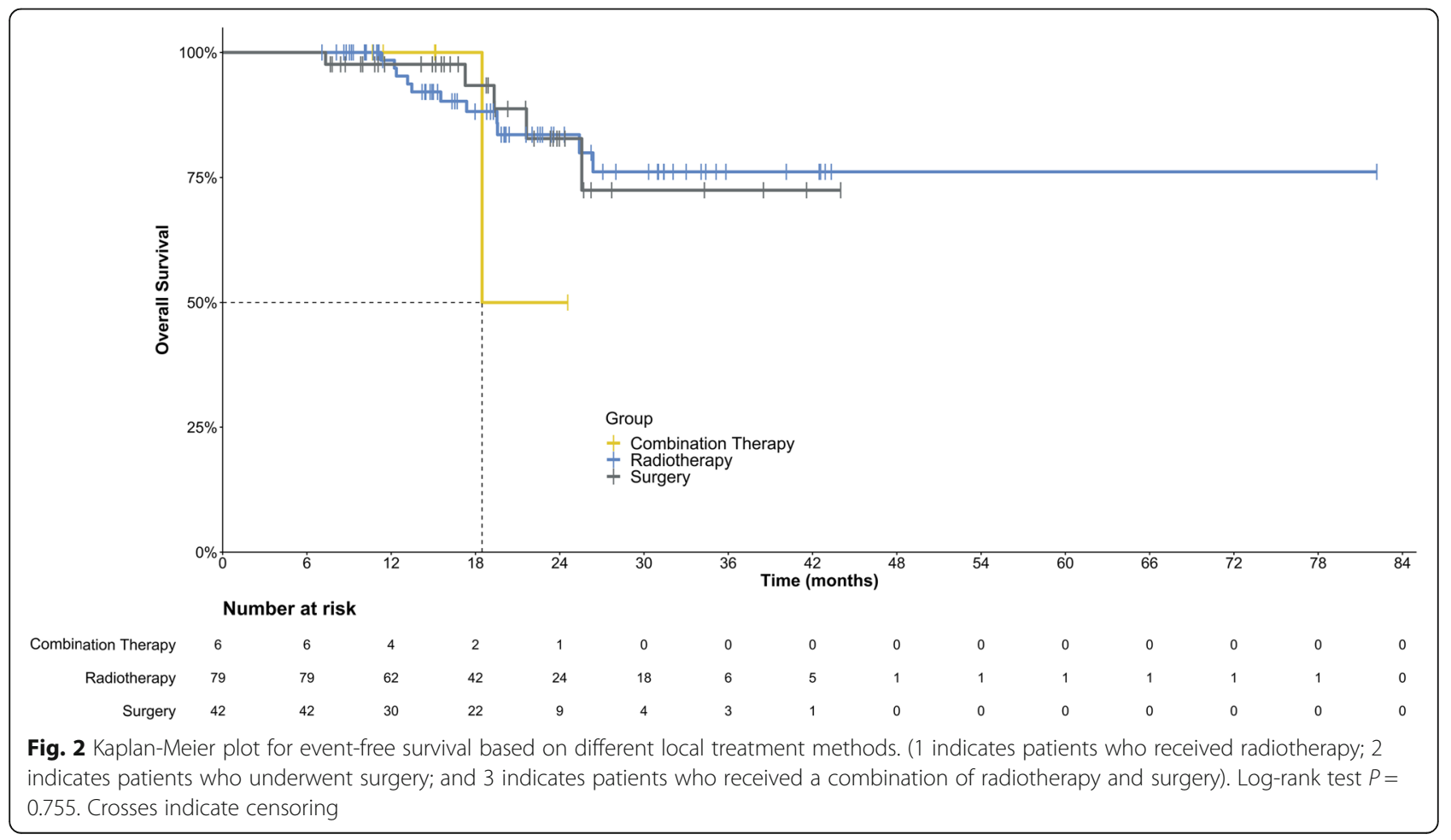




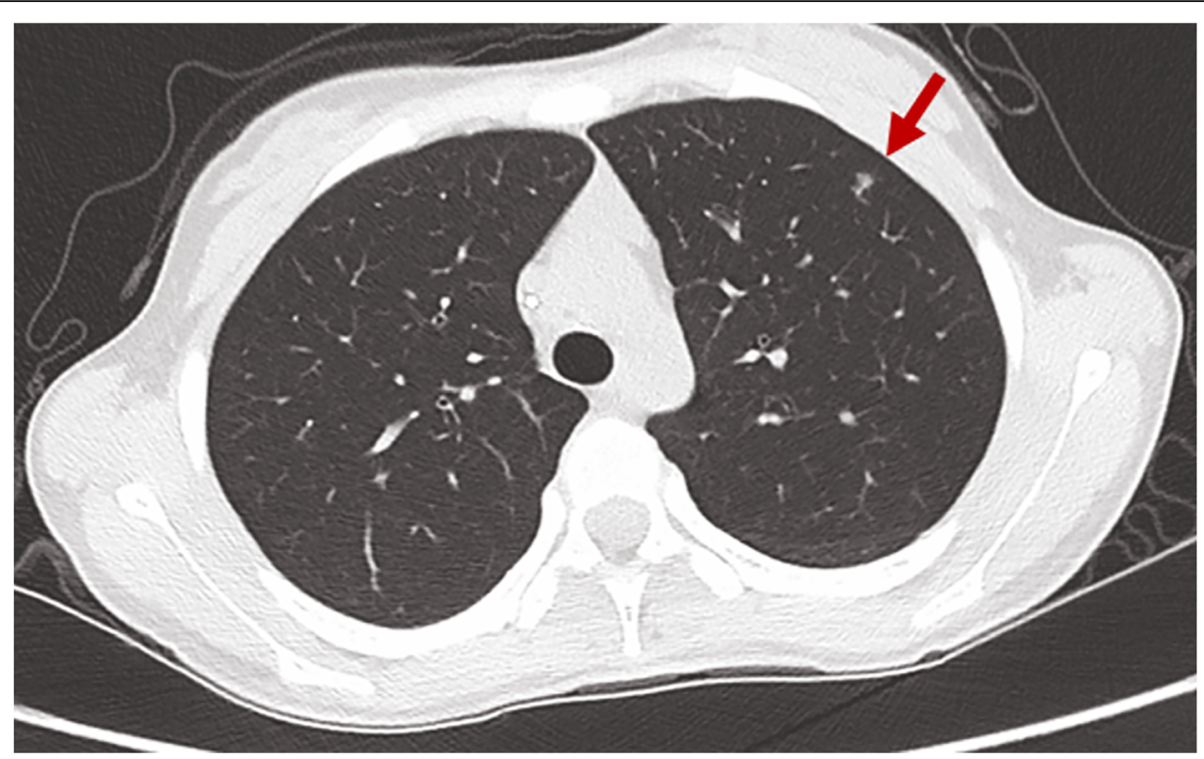

Fig. 3 A missing nodule shown on chest CT (red arrow) before metastasectomy. (It was not resected later. CT= computed tomography)

patients in our study and those who underwent open resection abroad. Our 2-year EFS rate was estimated to be $18.6 \%$ based on Kaplan-Meier analysis, which was not inferior to the Cooperative Osteosarcoma Study Group (COSS) results on recurrent osteosarcoma [12].

What was the prevalence of radiation-induced pneumonitis in these patients?

At follow-up, 62 of 86 (72.1\%) patients presented with pneumonitis on chest CT after radiation, 8 of whom (9.3\%) developed grade 2 pneumonitis according to the
Common Terminology Criteria for Adverse Events (CTCAE 5.0) and required steroids for palliation. One patient had pneumonitis that was so severe that she was administered steroid-included therapy for more than 3 months and later developed Pseudomonas aeruginosa infection. She ultimately died of disease progression or pyothorax (the data were not clear). The median time to the development of pneumonitis in our study was 3.5 (95\% CI: 1.0, 5.5) months after RT, with a duration of 7.2 (95\% CI: 6.9, 12.3) months (detailed information is presented in Table 4).

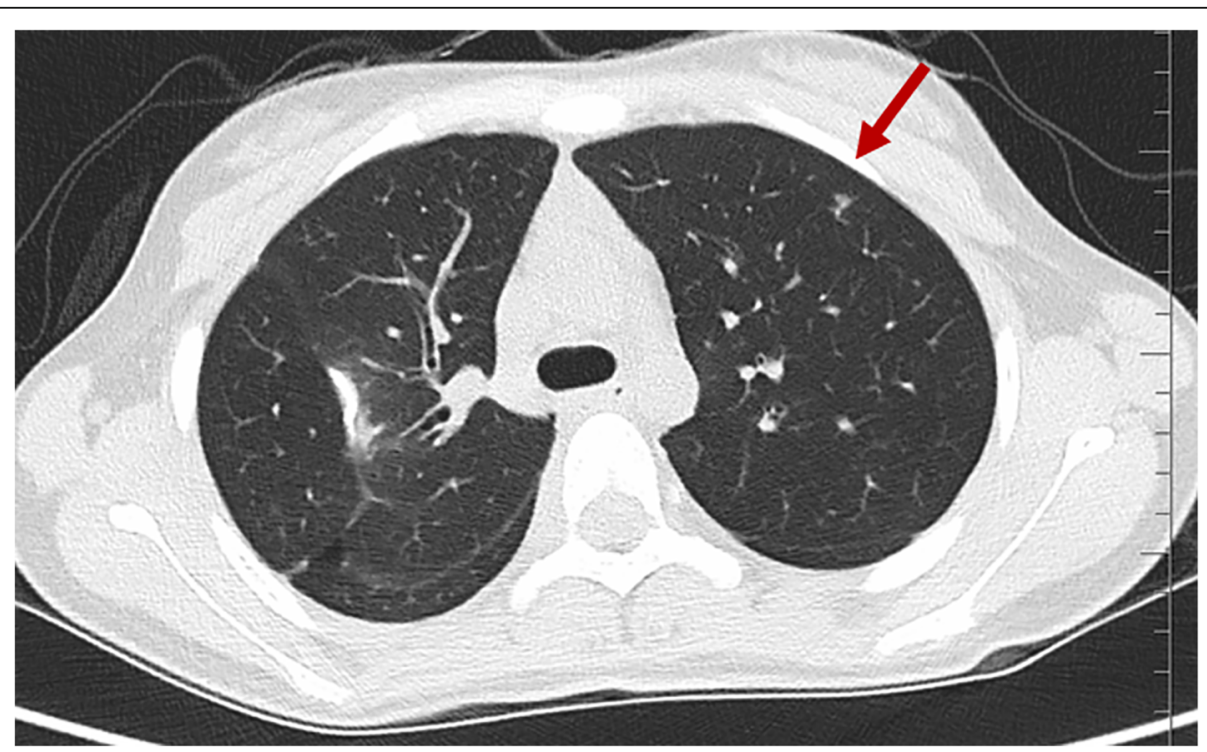

Fig. 4 A missing nodule shown on chest CT (red arrow) two weeks after metastasectomy. (A surgical scar can be observed on the right lobe of the lung. $C T=$ computed tomography) 


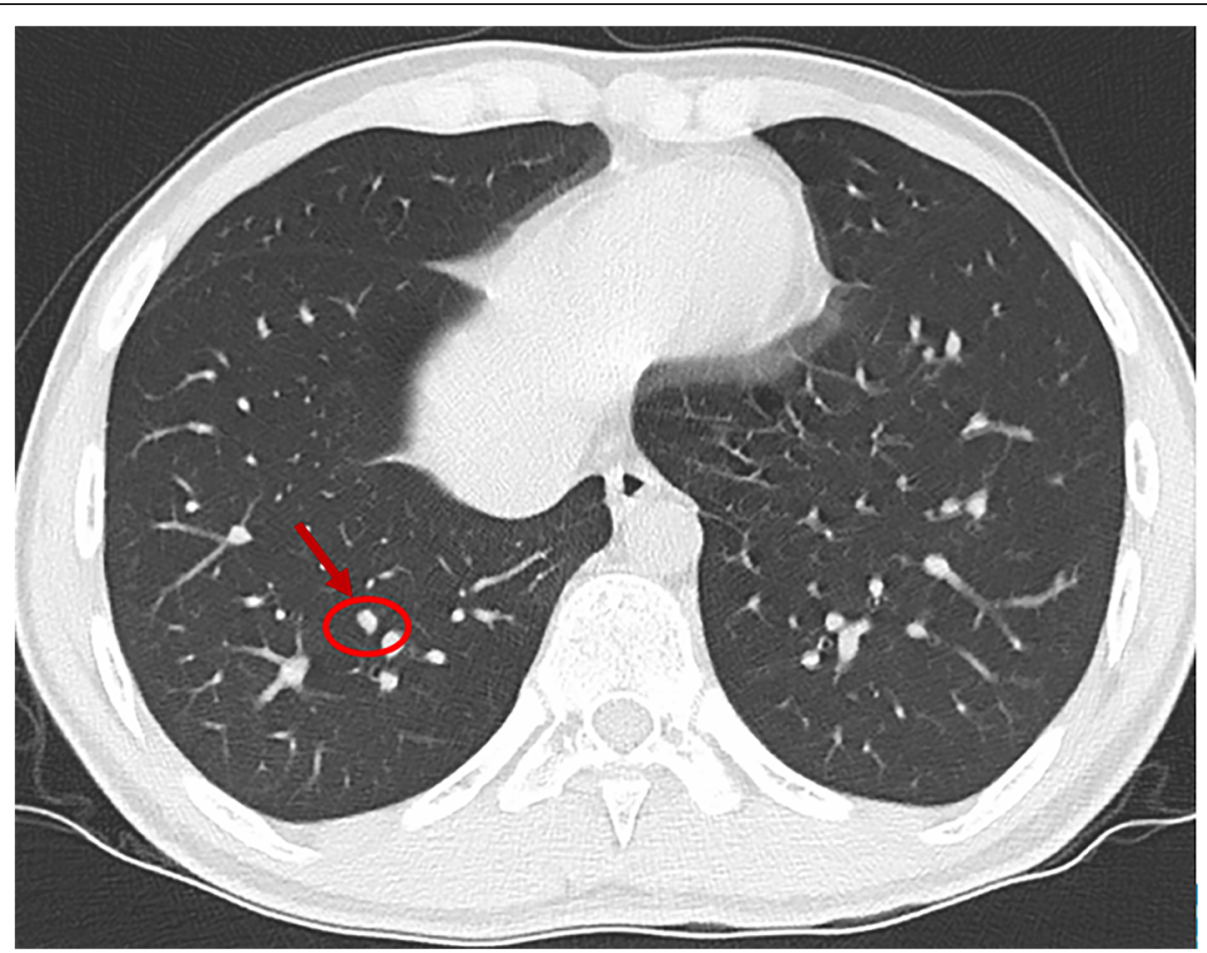

Fig. 5 A nodule less than $5 \mathrm{~mm}$ missing on chest $\mathrm{CT}$ (red circle) for radiation (This image was taken before hypofractionated radiotherapy. $\mathrm{CT}=$ computed tomography)

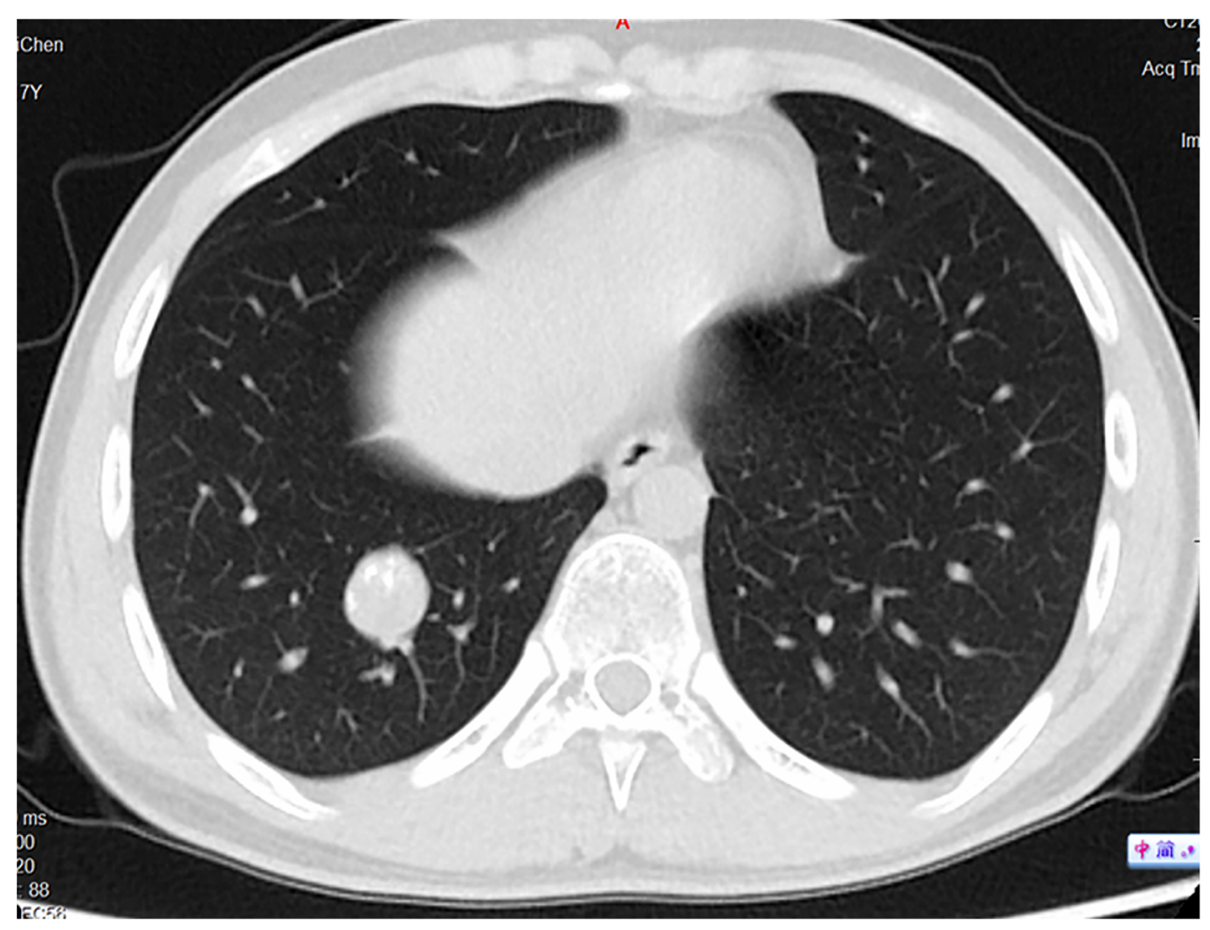

Fig. 6 A missing nodule progressed on chest CT (red circle) after radiation. (Eleven months later, this nodule, which had been missed for local therapy, became larger and obvious, and 3 new lesions also appeared on this patient's chest $C T$, all of which were later subjected to a second cycle of radiotherapy. At the last follow-up, the patient was still disease free. CT=computed tomography) 
Table 4 The incidence and duration of radiation-related pneumonitis ( $N=86)$

\begin{tabular}{lll}
\hline Items & Number of patients & Percentage \\
\hline Pneumonitis Grade ${ }^{a}$ & 62 & $72.1 \%$ \\
Grade 1 & 53 & $61.6 \%$ \\
Grade 2 & 8 & $9.3 \%$ \\
Grade 4 & 1 & $1.16 \%$ \\
$\begin{array}{l}\text { Onset of pneumonitis } \\
\text { (Median, 95\% Cl) }\end{array}$ & $3.5(1.1,5.5)$ months \\
$\begin{array}{l}\text { Duration of pneumonitis } \\
\text { (Median, 95\% Cl) }\end{array}$ & $7.2(6.9,12.3)$ months \\
Pneumonitis still not resolved until last follow up & 20 & $23.3 \%$ \\
\hline
\end{tabular}

${ }^{a}$ According to CTCAE 5.0

\section{Discussion}

This retrospective study showed that in China, the EFS rate for osteosarcoma patients with resectable pulmonary metastasis who received hypofractionated RT was comparable to that for those who received VATS during the same period and for those who underwent thoracotomies in published historical controls. The 12-month EFS rate in our total population was 35.6\% (95\% CI: $26.8,44.4 \%$ ), with $44.7 \%$ for VATS and $28.4 \%$ for radiation $(P=0.76)$. The COG conducted two prospective trials using small cohorts of patients with pulmonary recurrent osteosarcoma who received maintenance immunotherapy within 4 weeks after surgical complete remission, of which the 12-month EFS rates were 20\% (95\% CI: 10, 34\%) and 31\% (95\% CI: 17, 45\%), respectively, without a significant improvement in prognosis $[13,14]$. The COSS also previously evaluated the impact of patient-, tumour-, and treatment-related factors on the outcome in unselected patients with recurrent osteosarcoma recruited from a series of COSS studies between the end of 1979 and July 1998 [12]. At a median follow-up of 1.2 years for all patients and 4.2 years for survivors, the actuarial OS rates at 2,5 , and 10 years were $0.38,0.23$, and 0.18 , respectively, which, in our opinion, is slightly low, especially in terms of 2 -year OS. This might be due to the lack of more effective therapeutic schemes that were executed $30-40$ years ago. Our actual OS data are preliminary; thus, they were not comparable. However, from the Kaplan-Meier EFS curves of the COSS, we observed that the 2-year EFS rate was 19\% $(104 / 576)$ since relapse. Our data showed that the 2-year EFS rates were 15.4 and $10.9 \%$ for RT and VATS, respectively, indicating no inferiority to the COSS outcomes. Rizzoli et al. [6, 7, 15] conducted a series of studies that revealed the prognosis for high-grade osteosarcoma of the extremities metastatic to the lung, with a 5 -year EFS rate of 36\% for patients with localized disease who later relapsed and $9 \%$ for patients with resectable lung metastases at presentation; the difference was highly significant $(P<0.0001)$. However, they calculated
EFS from the beginning of treatment to relapse. It is interesting to note that in our study, the 5-year EFS rate was $18.6 \%$ for patients with initial metastasis (KaplanMeier estimated, when calculated from starting treatment to any event), which also showed no inferiority.

Sex, location, pathological subtypes, the number of pulmonary nodules, location at the unilateral pleura, local therapeutic methods, and lines of systemic therapy were irrelevant to prognosis in our study (Table 1). However, in our study, 3 of 127 (2.4\%) patients were older than 40 years and showed poorer EFS than those under 40 years $(P=0.025)$. Cox multivariate analysis indicated that no factor was correlated with outcomes. Thus, we hypothesize that currently, high-resolution chest $\mathrm{CT}$ ( $<3 \mathrm{~mm}$ per layer) can provide sufficient information for tiny pulmonary nodules to eradicate all lesions before RT or surgery, similar to thoracotomies [4, $5,7,12,15]$. However, we observed that $7.9 \%(52 / 657)$ of nodules were missed during follow-up; of which, for resection and for radiation, the missing rates were $15.0 \%$ $(15 / 100)$ and $6.6 \%$ (37/557), respectively. Table 3 also shows that only $1.3-4.8 \%$ of these untreated nodules alone would cause disease progression, while 12.7-14.3\% would relapse together with multiple new lesions, which indicated that these poor events were due to the progression of systemic disease and not to the untreated lesions. Furthermore, we observed that $45.2-62.0 \%$ of the cases of progression were not coupled with the local relapse of any existing lesion. Thus, we infer that with a local relapse rate as low as $5-15 \%$, disease progression is not because of local recurrence of pulmonary nodules, and such kinds of local therapy methods are adoptable.

However, radiation-induced lung injury (RILI), especially acute radiation pneumonitis, was observed in the majority of patients (72.1\%), with a median onset time of 3.5 (95\% CI: 1.1, 5.5) months and a duration of 7.2 (95\% CI: 6.9, 12.3) months. For cases involving $>10$ lesions that were larger than $4 \mathrm{~cm}$ and located beside the major bronchi, pneumonitis was so severe that we usually administered steroids to palliate symptoms. However, for 
RILI, the duration of corticosteroid use should always be 4 weeks or longer, which often causes a disruption in the anti-tumour immune microenvironment as well as secondary infections. Thus, currently, we choose VATS combined with RT for more complicated cases. Radiation is indicated for lesions located in sites that are difficult to manage during VATS. Surgeons often mark the chest CT scan 2 weeks after surgery for RT.

\section{Limitations}

This study had some limitations, including its small sample size and retrospective design. Selection bias was unavoidable in choosing patients who would receive local therapy. During the period in question, we had some general indications for the choice of surgery or radiation, as mentioned above. However, as a retrospective study, we are not certain that these indications were rigorously adhered to. The choice of local therapeutic method was deeply impacted by the patients' preferences. During our past experience, patients who had solitary lesions or less than 3 lesions and needed pathological confirmation conventionally received surgery, while those with more than 3 lesions or pulmonary nodules located in the central zone of the lung, which were deemed difficult to resect by VATS, usually received radiotherapy instead. Furthermore, the more times patients experienced pulmonary lesion relapse, the more the patients were inclined to choose radiation. Second, our follow-up time was not long enough to demonstrate OS and compare OS with that of historic controls. Third, we did not record in detail patients with secondary complete remission of pulmonary lesions or compare the outcomes with those who underwent CSR or CRR for the first time. Fourth, as only two Chinese sarcoma centres were involved in this study, our data might not completely reflect the current situation of osteosarcoma treatment in China. However, the local treatment of these pulmonary lesions was performed between June 2014 and July 2020 and in only two centres that might have used consistent systemic therapy and local therapy methods. Our experience should be shared and discussed when assessing local treatment options for such patients.

\section{Conclusions}

In patients with recurrent resectable pulmonary metastases from high-grade osteosarcoma that were deemed stable under systemic therapy, both hypofractionated RT and VATS can be considered adoptable options for local therapy methods because high-resolution chest CT can provide sufficient information on all these nodules. Therefore, the missing rate for these nodules could be relatively low, and we could mark and eliminate all the nodules. However, extra caution should be taken when using radiation to avoid severe pneumonitis.

\section{Abbreviations}

RT: Radiotherapy; OS: Overall survival; CT: Computed tomography; CSR: Complete surgical remission; VATS: Video-assisted thoracoscopic surgery; MDT: Multidisciplinary teamwork; SBRT: Stereotactic body radiotherapy; GKRS: Gamma Knife radiosurgery; PKUPH: Peking University People's Hospital; CRR: Complete radiated/metabolic remission;

Cl: Confidence interval.; PET: Positron emission tomography; EFS: Event-free survival; TKIs: Tyrosine kinase inhibitors; AOST: Osteosarcoma Protocols from Children's Oncology Group; COSS: Cooperative Osteosarcoma Study Group; CTCAE: Common Terminology Criteria for Adverse Events; RILI: Radiationinduced lung injury

\section{Supplementary Information}

The online version contains supplementary material available at https://doi. org/10.1186/s12885-021-08071-5.

Additional file 1 Appendix Fig. 1 Pictured is the updated osteosarcoma protocol used at Peking University People's HospitalOsteosarcoma (PKUPH-OS 02). DOX = doxorubicin; CDDP = cisplatin; IFO = ifosfamide; $V C R=$ vincristine; HD MTX = high-dose methotrexate.

\section{Acknowledgements}

We thank all the patients and their families for participating in this study. We also thank Dr. Danhua Shen for aiding in the pathological review of all pathologic slides.

\section{Authors' contributions}

Conception and design: LX, WG. Provision of study materials or patients: JX, ZZ, $H Z$, JG. Collection and assembly of data: LX, JX, XS, KL, XL1, ZZ, HZ. Pathological review of the slides in this trial: $K S$. Clinical evaluation of the study: $Y W, J G, L X$ and JX. Laboratorial work and molecular biological analysis of this study: KL, XL1 and XL2. Data analysis and interpretation: LX, JX, WG. Manuscript writing: LX. Accountable for all aspects of the work: $L X, J X, X L 1, Z Z, H Z, X S, K L, X L 2, K S, Y W$, $J G, W G$. All authors have read and approved the manuscript.

\section{Funding}

The authors of this study received funding from the Beijing Municipal Science \& Technology Project (No. Z181100001718054) and the Research and Development Fund of Peking University People's Hospital (Clinical Medicine $+X$ Cultivation Project, No. RDX2019-08) to support this study. The funders of the study had no role in the study design, data analysis, data interpretation, or writing of the report. All authors had access to the raw data, vouch for the completeness and accuracy of the data and analyses and approve the final version of the manuscript. The corresponding author had final responsibility for the decision to submit for publication

\section{Availability of data and materials}

We promised to maintain patient data confidentially. However, patient data will be made disguisedly available (including data dictionaries) for approved data sharing requests. Individual data that underlie the results reported in this article will be shared after de-identification and normalization of information (text, tables, figures, and appendices). The statistical analysis plan will also be available upon request. Anonymised data will be available beginning 3 months after and ending 2 years after publication of this article to researchers after a methodological review of the proposed analysis plan by the directors of the Musculoskeletal Tumor Center of Peking University People's Hospital or Peking University Shougang Hospital. Proposals should be directed to xie.lu@hotmail.com. To gain access, data requestors will need to sign a data access agreement.

\section{Declarations}

\section{Ethics approval and consent to participate}

This study was retrospectively registered in the Medical Ethics Committee of Peking University People's Hospital and Peking University Shougang Hospital. The trial registration number is 2020PHB060-02, and the date of registration is September 11th, 2020. It has also been registered online at Clinicaltrials. gov under identifier NCT04546243. No consent forms were needed because this was a retrospective study. 


\section{Consent for publication}

Not Applicable.

\section{Competing interests}

The institution of one or more of the authors ( $L X, J X, X L 1, Z Z, X S, K L, X L 2, K S$, YW, JG, WG, and HZ) received funding from the Beijing Municipal Science \& Technology Project (No. Z181100001718054) and the Research and Development Fund of Peking University People's Hospital (Clinical Medicine +X Cultivation Project, No. RDX2019-08).

\section{Author details}

'Musculoskeletal Tumor Center, Peking University People's Hospital, No. 11 Xizhimen South Street, Xicheng District, Beijing, PR China. ${ }^{2}$ Department of Thoracic Surgery, Peking University People's Hospital, No. 11 Xizhimen South Street, Xicheng District, Beijing, PR China. ${ }^{3}$ Department of Radiotherapy, Peking University Third Hospital, No. 49 North Garden Rd., Haidian District, Beijing, PR China. ${ }^{4}$ Pathology Department, Peking University People's Hospital, No. 11 Xizhimen South Street, Xicheng District, Beijing, PR China. ${ }^{5}$ Endocrinology Department, Peking University Shougang Hospital, No. 9 Jinyuanzhuang Road, Shijingshan District, Beijing, PR China. ${ }^{6}$ Surgical Oncology, Peking University Shougang Hospital, No. 9 Jinyuanzhuang Road, Shijingshan District, Beijing, PR China.

Received: 3 December 2020 Accepted: 19 March 2021

Published online: 31 March 2021

\section{References}

1. Smeland S, Bielack SS, Whelan J, Bernstein M, Hogendoorn P, Krailo MD, et al. Survival and prognosis with osteosarcoma: outcomes in more than 2000 patients in the EURAMOS-1 (European and American osteosarcoma study) cohort. Eur J Cancer. 2019;109:36-50. https://doi.org/10.1016/j.jca.2 018.11.027.

2. Luetke A, Meyers PA, Lewis I, Juergens H. Osteosarcoma treatment - where do we stand? A state of the art review. Cancer Treat Rev. 2014;40(4):523-32. https://doi.org/10.1016/j.ctrv.2013.11.006.

3. Arshi A, Sharim J, Park DY, Park HY, Yazdanshenas H, Bernthal NM, et al. Prognostic determinants and treatment outcomes analysis of osteosarcoma and ewing sarcoma of the spine. Spine J. 2017;17(5):645-55. https://doi. org/10.1016/j.spinee.2016.11.002.

4. Steliga $\mathrm{M}$, Vaporciyan $\mathrm{A}$. Surgical treatment of pulmonary metastases from osteosarcoma in pediatric and adolescent patients. In: Jaffe N, Bruland O, Bielack S, editors. Pediatric and adolescent osteosarcoma cancer treatment and research. Boston: Springer; 2009. p. 185-201.

5. Bhattasali O, Vo AT, Roth M, Geller D, Randall RL, Gorlick R, et al. Variability in the reported management of pulmonary metastases in osteosarcoma. Cancer Med. 2015;4(4):523-31. https://doi.org/10.1002/cam4.407.

6. Briccoli A, Rocca M, Salone M, Guzzardella GA, Balladelli A, Bacci G. High grade osteosarcoma of the extremities metastatic to the lung: long-term results in 323 patients treated combining surgery and chemotherapy, 19852005. Surg Oncol. 2010;19(4):193-9. https://doi.org/10.1016/j.suronc.2009.05. 002.

7. Bacci G, Rocca M, Salone M, Balladelli A, Ferrari S, Palmerini E, et al. High grade osteosarcoma of the extremities with lung metastases at presentation: treatment with neoadjuvant chemotherapy and simultaneous resection of primary and metastatic lesions. J Surg Oncol. 2008;98(6):415-20. https://doi.org/10.1002/jso.21140.

8. Brown LC, Lester RA, Grams MP, Haddock MG, Olivier KR, Arndt CAS, et al. Stereotactic body radiotherapy for metastatic and recurrent ewing sarcoma and osteosarcoma. Sarcoma. 2014;2014:418270.

9. Bowden G, Kano H, Caparosa E, Park SH, Niranjan A, Flickinger J, et al. Gamma knife radiosurgery for the management of cerebral metastases from non-small cell lung cancer. J Neurosurg. 2015;122(4):766-72. https://doi. org/10.3171/2014.12.JNS141111.

10. Bowden G, Kano H, Tempel ZJ, Caparosa E, Monaco E, Niranjan A, et al. Gamma knife radiosurgery for management of cerebral metastases from esophageal carcinoma. J Neuro-Oncol. 2014;118(1):141-6. https://doi.org/1 0.1007/s11060-014-1408-3.

11. De Rose F, Franceschini D, Reggiori G, Stravato A, Navarria P, Ascolese AM, et al. Organs at risk in lung SBRT. Physica Med. 2017;44:131-8. https://doi. org/10.1016/j.ejmp.2017.04.010.
12. Kempf-Bielack B, Bielack SS, Jürgens H, Branscheid D, Berdel WE, Exner GU, et al. Osteosarcoma relapse after combined modality therapy: an analysis of unselected patients in the cooperative osteosarcoma study group (COSS). J Clin Oncol. 2005;23(3):559-68. https://doi.org/10.1200/JCO.2005.04.063.

13. Carola AS, Arndt EA. A phase II study of aerosolized GM-CSF (NSC\# 613795, IND\# 11042) in patients with first pulmonary recurrence of osteosarcoma. In: ASCO Conference: Children's Oncology Group; 2008. p. AOST0221.

14. Hingorani P, Krailo MD, Buxton A, Hutson PR, Davis J, Janeway KA, et al. A phase 2 study of human-mouse chimeric anti-disialoganglioside monoclonal antibody ch14.18 (Dinutuximab, NSC\# 764038, IND\# 4308) in combination with sargramostim (GM-CSF) in patients with recurrent osteosarcoma. In: ASCO Conference: Children's Oncology Group; 2020. p. AOST1421.

15. Bacci G, Briccoli A, Rocca M, Ferrari S, Donati D, Longhi A, et al. Neoadjuvant chemotherapy for osteosarcoma of the extremities with metastases at presentation: recent experience at the Rizzoli institute in 57 patients treated with cisplatin, doxorubicin, and a high dose of methotrexate and ifosfamide. Ann Oncol. 2003;14(7):1126-34. https://doi.org/10.1093/annonc/ mdg286.

\section{Publisher's Note}

Springer Nature remains neutral with regard to jurisdictional claims in published maps and institutional affiliations.
Ready to submit your research? Choose BMC and benefit from:

- fast, convenient online submission

- thorough peer review by experienced researchers in your field

- rapid publication on acceptance

- support for research data, including large and complex data types

- gold Open Access which fosters wider collaboration and increased citations

- maximum visibility for your research: over $100 \mathrm{M}$ website views per year

At BMC, research is always in progress.

Learn more biomedcentral.com/submissions 